\title{
Correction
}

\section{Correction: Marrocco et al., Anxiety-Like Behavior of Prenatally Stressed Rats Is Associated with a Selective Reduction of Glutamate Release in the Ventral Hippocampus}

In the article "Anxiety-Like Behavior of Prenatally Stressed Rats Is Associated with a Selective Reduction of Glutamate Release in the Ventral Hippocampus” by Jordan Marrocco, Jérôme Mairesse, Richard Teke Ngomba, Viviana Silletti, Gilles Van Camp, Hammou Bouwalerh, Maria Summa, Anna Pittaluga, Ferdinando Nicoletti, Stefania Maccari, and Sara Morley-Fletcher, which appeared on pages 17143-17154 of the November 28, 2012 issue, the authors regret a typographical error in the molecular weights of the immunoblots in Figure 2D. This error is not reflected in the immunoblot quantification and presented bands, nor in the figure legend, neither in the reference of the antibodies, or anywhere else in the paper. Thus, interpretation and conclusions of the study are not affected. The correct Figure 2 is shown below.

A

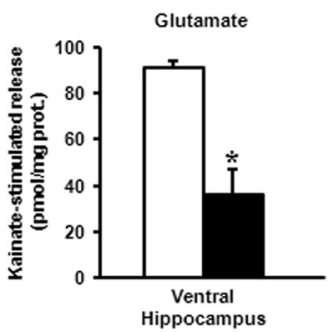

D

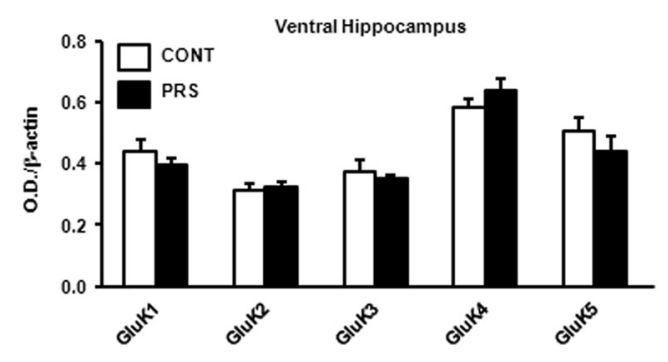

B

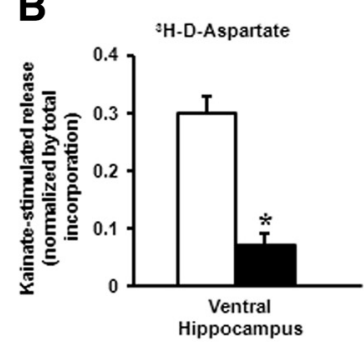

C

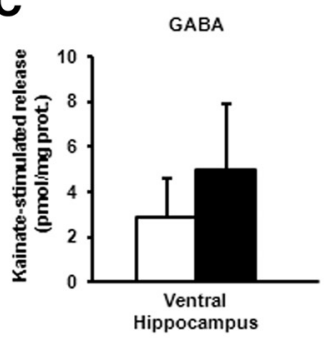

CONT PRS

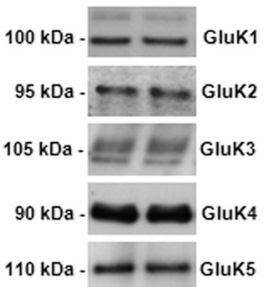

Kainate-induced EEG alteration: score levels

\section{E}
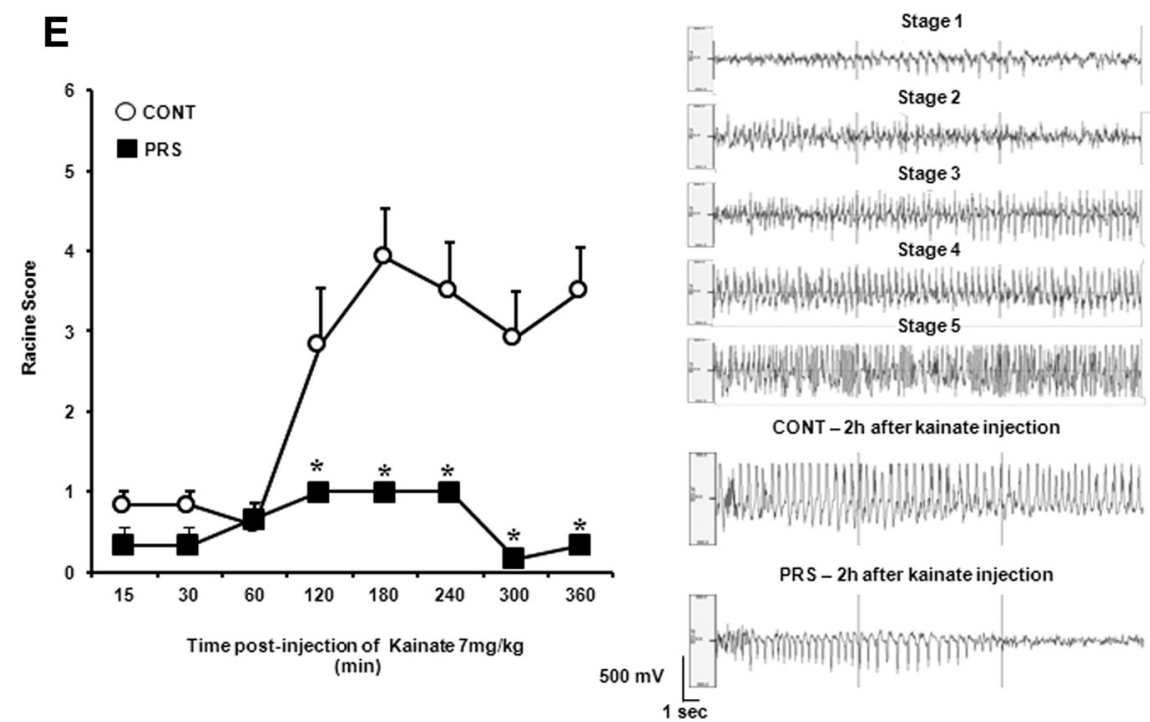

Figure 2. 